\title{
Session 8: Molecular biology - II
}

\author{
Thursday 7 October 2004. Moderators: Jim Larrick and Sachev S. Sidhu
}

\section{$[16.00-16.20]$}

\section{Optimising antibody drugs by ribosome and phage display \\ Ralph Minter \\ Lead Optimisation Group, Cambridge Antibody Technology}

Over the last ten years phage display has been employed for the potency optimisation of many antibodies which have progressed into clinical development. More recently, ribosome display has emerged as a powerful alternative to phage display for antibody optimisation. In this study we have analysed in-house data from the optimisation of 30 antibodies by phage and/or ribosome display to compare the efficacy of these display technologies. Data will be presented to show that both display technologies can improve potency over 10,000 -fold and furthermore that ribosome display has the ability to search wider areas of sequence space for benefical mutations. The optimisation of an anti-IL13 antibody, which is now in pre-clinical development, will be covered in greater detail as an example of how the optimisation process can also yield useful information about the antigen binding site.

\section{[16.20-16.40]}

\section{Identification of potent and specific membrane} type serine protease inhibitors using phage display Andrew E. Nixon, Rene Hoet, Nicole Hoebers and Que Nguyen

Dyax Corp. 300 Technology Square, Cambridge, MA 02339, Susanne Anderson \& Ed Madison, Dendreon San Diego LLC, 3030 Science Park Road, San Diego, CA, USA

Membrane type serine proteases (MTSPs) are type II membrane proteins, the expression of which is upregulated in tumor tissues. As the protease domains of MTSPs are extracellular, this has led to the proposal that the proteases may act either to activate sig- naling molecules and so stimulate proliferation or to degrade proteins of the extracellular matrix to facilitate metastasis. MTSPs therefore represent potential targets for cancer therapeutics. Two novel MTSPs, Endotheliase-1 and Endotheliase-2 have been cloned and the serine protease domains overexpressed and purified. To identify inhibitors of their serine protease activity, we used the protease domains of endotheliase-1 and endotheliase- 2 in a series of phage display library selections using peptide, Kunitz domain and antibody libraries. From the antibody library selections we identified a panel of antibodies with Kd's in the picomolar to nanomolar range before affinity maturation. Here we will describe the selection approach, characterization of the protease inhibitors identified from the libraries and the application of the antibodies to further study of the expression of these protease targets.

\section{[16.40-17.00]}

Generation of a recombinant human anti-sperm antibody, RASA: Implications as an intravaginal spermicidal agent

Kimberly D. Fralix, Bingfang Xu, Elizabeth J. Norton, Alan B. Diekman, Michael A. Coppola and John C. Herr

Center for Research in Contraceptives and Reproductive Health, University of Virginia Medical Center, Charlottesville, VA, USA

Current intravaginal spermicidal products contain detergent-based active ingredients, such as Nonoxynol9, which have been shown to have deleterious effects on the vaginal epithelium, thereby compromising a natural barrier against pathogenic invasion and increasing susceptibility to sexually transmitted diseases. The World Health Organization and the US FDA have released statements in support of these findings that frequent use of these products causes vaginal irritation which may increase the risk of HIV/STI transmission. As an alternative to these detergent-based products, an 
antibody-mediated spermicidal/spermistatic agent is in development.

A monoclonal antibody, S19, derived from immunization of mice with human sperm extracts exhibits significant contraceptive effects in laboratory tests. S19 recognizes a reproductive tract-specific antigen localized on the sperm surface. When bound to this antigen, S19 completely agglutinates and immobilizes sperm, inhibits sperm-zona pellucida tight binding, and inhibits sperm penetration of cervical mucus in vitro making it an ideal contraceptive candidate. In an effort to produce a clinically useful model, the full-length antibody was shortened to a $\mathrm{ScFv}$ (single chain variable fragment) for ease of production and humanized to reduce the likelihood of HAMA (human anti-mouse antibody) responses.

The ScFv of S19, termed RASA (Recombinant AntiSperm Antibody), was generated by cloning the variable regions of the heavy and light chains of S19 from hybridoma cells and joining them with a polypeptide linker (Gly3Ser)4. RASA was expressed in E. coli and shown to maintain the same inhibitory properties as the parent molecule, S19, namely complete sperm agglutination and immobilization in vitro, thus establishing proof-of-principle. RASA was then humanized by overlap extension PCR which allowed simultaneous site-directed mutagenesis of amino acids in the variable region framework to resemble those typically found in human IgG. Overlapping oligonucleotides of approximately 85-95 bp spanning the entire RASA sequence (760 bp) were used in a two-step PCR process which first annealed and extended the Fv-coding regions of the heavy and light chains independently. The two segments were then connected via the linker (Gly3Ser) 4 and extended by short outside primers to produce the full-length construct.

Platforms for large-scale production are currently being investigated. Large quantities of soluble protein have been successfully produced in Yeast (P. Pastoris), although activity has been compromised. Optimization of growth conditions and/or refolding procedures may allow for production of the active conformation. Bioengineering of tobacco plants (N. tabacium) has produced soluble, active RASA and with optimization of scale-up production and purification schemes this system should yield large quantities of RASA in a costeffective manner. Further development of these production systems is ongoing.

This research provides a model for spermicides based on anti-sperm antibodies and, to the best of our knowledge, has developed the first active recombinant an- tibody to the surface of human sperm. Conceivably, RASA will serve as a prototype for a generation of spermicides without detergent properties to reduce the risks of HIV/STD transmission to women who are already at high risk.

\section{[17.00-17.20]}

\section{Human immunoagents anti-ErbB2-positive carcinomas \\ Claudia De Lorenzo, Renata Piccoli and Giuseppe \\ D'Alessio \\ Department of Biological Chemistry University of \\ Naples Federico II, Naples, Italy}

We have isolated [1] a human anti-ErbB2 scFv from a phage display library [2]. This scFv, dubbed Erbicin, binds with high affinity ( $\mathrm{Kd} 4 \mathrm{nM}$ ) to ErbB2-positive cells, it is selectively internalized, and inhibits target cells growth. Taking advantage of the availability of Erbicin, we have used it to construct two fusion immunoagents directed to ErbB2 expressing cells.

The first is an immunoRNase (hErb-RNase), in principle derived from the immunotoxin concept, but hardly toxic and immunogenic as a classic immunotoxin. hErb-RNase is in fact an immuno-pro-toxin, as it is made up of Erbicin fused to a human, per se non-toxic RNase (HP-RNase). HP-RNase, an RNase ubiquitous in human organisms, in contrast with the powerful bacterial or plant toxins so far fused in the immunotoxin constructs, can exert cytotoxic effects only if and when it is internalized in target cells. Our data show that hErb-RNase is selectively internalized by, and is selectively cytotoxic for, ErbB2 overexpressing cells. Recent results have shown that hErb-RNase is strongly active also in vivo on tumors induced in mice by inoculation of breast carcinoma cells [3].

The second Erbicin derived immunoagent is an engineered antibody in which the Erbicin $\mathrm{scFV}$ is fused to a human IgG1 Fc. As the fusion antibody (called Erb-hcAb) is short of the $\mathrm{CH} 1$ and CL sequences, it constitutes a human, bivalent anti-ErbB2 antibody, but it is compact, i.e. with a reduced size compared to a full IgG [4]. We found that Erb-hcAb displays a selective cytotoxic activity in vitro and in vivo on carcinoma cells that overexpress ErbB2. The compact molecular size of Erb-hcAb is likely to provide for an efficient tissue penetration, compared to full-size anti-ErbB2 antibodies. Yet, compared to small immunoagents, such as $\mathrm{scFv}$ or Fab fragments, it should have a longer serum half-life. 
Our first data on the mechanism of antitumor action of these anti-ErbB2 immunoagents have indicated that they affect phosphorylation of ErbB2 in different manners. The compact, bivalent antibody is able to induce homodimerization of ErbB2, and likely its lysosomal degradation, whereas the monovalent $\mathrm{scFv}$ and immunoRNase inhibit its hetero-dimerization with other ErbB receptors, which consequently lowers the oncogenic effects.

\section{References}

[1] C. De Lorenzo, D.B. Palmer, R. Piccoli, M.A. Ritter and G. D'Alessio, A new human antitumor immunoreagent specific for ErbB2, Clin. Cancer Res. 8 (2002), 1710-1719.

[2] A.D. Griffiths, S.C. Williams, O. Hartley et al., Isolation of high affinity human antibodies directly from large synthetic repertoires, EMBO J 13 (1994), 3245-3260.

[3] C. De Lorenzo, A. Arciello, R. Cozzolino, D.B. Palmer, P. Laccetti, R. Piccoli and G. D'Alessio, A Fully Human Anti-tumor ImmunoRNase Selective for ErbB2-Positive Carcinomas, Cancer Res (2004), in press.

[4] C. De Lorenzo, A. Tedesco, G. Terrazzano, R. Cozzolino, P. Laccetti, R. Piccoli and G. D'Alessio, A human, compact, fully functional anti-ErbB2 antibody as a novel antitumor agent, submitted.

\section{[17.20-17.50]}

Fully human antibodies for the treatment of severe diseases derived from the human combinatorial antibody library HuCAL ${ }^{\circledR}$ GOLD

Thomas von Rüden

MorphoSys AG, Lena-Christ-Str. 48, D-82152

Martinsried, Germany

Administrative contact: Ilse Luig: E-mail:

luig@morphosys.com

MorphoSys' proprietary Human Combinatorial Antibody Library, HuCAL ${ }^{\circledR}$ GOLD, has proven to be a robust platform for the generation of high-quality human antibodies against a variety of different antigens. It is now widely used by top tier pharmaceutical and biotechnology companies for target research and, in particular, for the generation of therapeutic antibodies for the treatment of various pathologies, including cancer (solid tumors, as well as hematological malignancies), Alzheimer's disease, cardiovascular disease and inflammatory disorders.

In our own proprietary discovery programs, we have generated the first fully human antibodies, which are several in number, against ICAM-1 for the treatment of inflammatory diseases. Comprehensive in-vitro profiling of the human antibodies, which had not been optimized, revealed an efficacy profile comparable to that of murine antibody BIRR-1. Initial in-vivo experiments demonstrated very good efficacy in a psoriatic skin transplant model. Moreover, in contrast to BIRR1, none of the HuCAL-derived antibodies caused activation of neutrophils.

We also have isolated antibodies targeting CD38 for the treatment of hematological malignancies. These HuCAL-derived antibodies have unique properties that differentiate them positively from previously generated murine antibodies against such targets. Further, these antibodies exhibit very good efficacy in killing CD38-positive myeloma cell lines, as well as primary myeloma cells.

Most recent developments from our own and partnered programs will be presented.

\section{[17.50-18.20]}

\section{Design and characterisation of multivalent} antibody molecules

Peter J. Hudson ${ }^{1,2}$, B.E. Power ${ }^{1}$, O. Dolezal ${ }^{1,2}$, J. Atwell $^{1,2}$, K. Tahtis ${ }^{3}$, F.T. Lee ${ }^{3}$, A.M. Scott ${ }^{3}$, J.

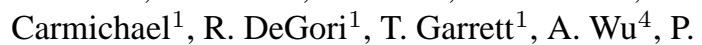

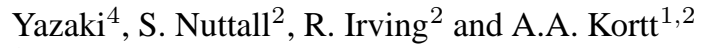
${ }^{1}$ CSIRO Health Sciences and Nutrition

${ }^{2}$ CRC for Diagnostic Technologies, 343 Royal Parade, Parkville, Victoria, Australia, 3052

Tel.: +61 3 96627100; Fax: 7101

${ }^{3}$ Ludwig Institute for Cancer Research, Austin Repatriation Hospital, Heidelberg, Victoria, Australia

${ }^{4}$ Beckman Research Institute, City of Hope, Duarte, California, USA

Based on our scFv crystal structures, we have designed numerous point mutations to increase the affinity of the antigen binding surface $[1,2]$. However, functional affinity in $\mathrm{scFv}$ is more easily achieved by multimerisation, for example by careful linker design to direct the formation of diabodies, triabodies or tetrabodies $[3,4]$. Importantly, for cancer targeting and other in vivo applications, the higher avidity and larger size of scFv multimers ( $\sim 60-100 \mathrm{kDa}$ in size) offers several important advantages in pharmacokinetics [5]. Multimeric scFvs (diabodies and triabodies) are better than the parent antibody for imaging applications due to both improved tumor penetration and faster blood clearance. Precise structural designs of the linker length between the $\mathrm{V}$-domains from 5 to 0 residues forced the structure from dimers $(\sim 60 \mathrm{kDa})$ to either trimers $(\sim 90 \mathrm{kDa})$ or tetramers $(\sim 120 \mathrm{kDa})$ depending on V-domain orientation $[3,4]$. The binding valency was confirmed by elec- 
tron microscope imaging of single molecules $[3,4]$. Diabody crystal structures also identified unique residues in the associating FV surfaces that modulate the preferred association into dimers, trimers or tetramers [3].

Most recently, we have solved the diabody crystal structures of two cancer targeting antibodies. The first was the anti-CEA (Carcino-embryonic antigen) monoclonal antibody T84.66 that had previously been used for targeting colorectal carcinomas [6]. The diabody crystal structure at $2.6 \AA$ showed that the associations between Fv modules in this VL to VH diabody are different to other diabodies and provide insights into the preferred linker length, orientation and stability. The second diabody was the humanised anti-Lewis Y monoclonal antibody (hu3S193), recently evaluated for targeting epithelial tumors [7]. The structure was solved to $2.6 \AA$ both with and without the target antigen, Lewis$y$ tetrasaccharide. The pharmacokinetics of ${ }^{111}$ Inlabelled diabody compared to conventional $\left.\mathrm{F}(\mathrm{ab})_{2}\right)_{2}$ in MCF-7 BALB/c nude mouse xenografts showed excellent tumour:blood ratios at 8 hours of 20:1 and 8:1 respectively $[7,8]$.

To complement the current range of peptide and anti- body repertoires, we have constructed display libraries of novel antibody-like V-domains [9-11]. These Vdomains form efficient scaffolds for the presentation and selection of constrained polypeptides displayed as long surface loop structures. Importantly, V-domains can penetrate clefts in the antigen (enzymes, viral capsids and cell-surface receptors) and therefore target both refractory antigens and immunosilent cavities.

\section{References}

[1] Malby et al., J. Mol Biol. 279 (1998), 901-910.

[2] Dougan et al., Protein Engineering 11 (1998), 65-74.

[3] Dolezal et al., Protein Engineering 13 (2000), 565-574; (2002) Protein Engineering in press.

[4] Todorovska et al., J. Immunol. Methods. 248 (2001), 47-66.

[5] Hudson and Souriau, Expt Opin. Biol. Therapy 1 (2001), 845-855. (2002) Nature Medicine in press.

[6] Wu et al., Proc. Natl. Acad. Sci. USA. 97 (2000), 849. Carmichael et al., J. Mol Biol (2002), in press.

[7] Tahtis et al., Clin. Cancer Res. 7 (2001), 1061-1072.

[8] Power et al., Cancer. Immunol. Immunother. 50 (2001), 241-250.

[9] Nuttall et al., Proteins 36 (1999), 217-227.

[10] Nuttall et al., Curr. Pharma. Biotech 1 (2000), 253-263.

[11] Irving et al., J. Immunol. Meth. 248 (2001), 31-46. 